\title{
Design of a Well-Defined Poly(Dimethylsiloxane)-Based Microbial Nanoculture System
}

\author{
Huda Usman, ${ }^{l}$ Shanna-Leigh Davidson, ${ }^{1}$ Nithil H. Manimaran, ${ }^{1}$ Jenna T. Nguyen, ${ }^{l}$ Aïssatou Bah, ${ }^{l}$ \\ Rishabh Seth, ${ }^{2}$ Eric Beckman, ${ }^{1}$ Tagbo H.R. Niepa ${ }^{1,2,3,4,5 *}$
}

${ }^{1}$ Department of Chemical and Petroleum Engineering, ${ }^{2}$ Department of Bioengineering, ${ }^{3}$ Department of Civil and Environmental Engineering, ${ }^{4}$ Department of Mechanical Engineering and Materials Science, ${ }^{5}$ Center for Medicine and the Microbiome, University of Pittsburgh, Pittsburgh, PA 15261, United States

\section{E-mail: tniepa@pitt.edu}

Keywords: poly(dimethylsiloxane) membranes, microfluidics, microbial cultures, biofilm, growth dynamics, microbial community

Organosilanes contain hydrocarbon-like backbones, allowing them to react with silicone-based agents in the presence of a catalyst and polymerize into membranes with tunable transport and mechanical properties. Owing to their high hydrophobicity, Poly(dimethylsiloxane) (PDMS) membranes, and more particularly, Sylgard ${ }^{\circledR} 184$, have been used for applications including drug delivery, gas separation, and microfluidics fabrication. However, the undefined composition of the material and its ability to leach out uncured oligomers make its functionalization and usage challenging for many biological applications. This article presents the design of a novel culture system generated using PDMS-based membranes to study microbial dynamics. The nanoliter microbial culture system that is referred to as "nanoculture" serves to encapsulate and grow microbes in semipermeable membranes. The mechanical properties of the membranes are investigated through osmotic annealing to determine the ability of the nanocultures to withstand high shear stress similar to environmental conditions while maintaining transport properties essential to microbial communication and growth. The present study lays the 
foundation for a novel microbial culture system that would enable the cultivation of microorganisms in environments other than laboratory conditions.

\section{Introduction}

The void in antibiotic discovery that has plagued the infection control community for the last three decades could be solved if one overcomes the inability to culture a majority fraction of microorganisms existing in myriad ecosystems. ${ }^{1}$ Like Penicillium notatum in the discovery of penicillin, many microorganisms harbor the key for naturally occurring bioactive molecules that could help design new classes of antibiotics and address the current crisis. Unfortunately, the unculturability of the many viable species that spread from environmental samples, such as soil and marine, to the human microbiome remains an extensive problem that impedes the discovery potential of novel penicillin-like compounds. The phenomenon of "viable but unculturable" microorganisms, popularly termed "microbial dark matter,"2 has prodded a new way of thinking regarding traditional culturing methods. Novel culturing methods are now required to close the gap between the species known to exist, due to omics studies, and the few that are currently culturable with conventional methods. The problems underlying microbial unculturability are founded on the lack of understanding and knowledge of how to fully simulate their native microenvironments. ${ }^{3}$ Several factors can be manipulated when trying to simulate a native environment, such as $\mathrm{pH}$, temperature and osmotic pressures, all of which when combined, result in a complex matrix of tunable variables. ${ }^{4}$ The challenge of culturing these recalcitrant microorganisms has resulted in innovative technologies, with some groups reaching a $40 \%$ increase in diversity of isolated cultures, as compared to that of traditional plating. ${ }^{5}$

One successful avenue for exploring the diverse population of unculturable microbes is through diffusion or isolation chambers such as the iChip. ${ }^{5}$ The method consists in sequestering microbial isolates in a device made of micromachined chambers, in which nutrients and agar can be introduced. ${ }^{6}$ 
The device can be incubated in situ to expose the isolates to their original environment. Because the cells are confined in the microchambers and separated from their surrounding via semipermeable membranes, they can receive metabolites and growth factors diffusing into the microchambers, and grown without having to compete for nutrients and space against other organisms in the vicinity of the device. The technique allows the cultivation of hundreds of operational taxonomic units from soil and marine isolates, corresponding to an improvement by $\sim 40 \%$ over standard culture plate methods. ${ }^{5}$

With recent advances in microfluidics, and more specifically in their high-throughput nature, it is hoped that one can accelerate the isolation and culturing processes of new microbes. Microfluidics offers complementary advantages to advance the culturing of microbes in diffusion/isolation chambers. The technique takes the beneficial aspects of other methods and brings them into the lab using a workable scale. Now, the idea of bringing an entire environment into the lab become less laborious and more feasible, especially if one leverages microscale technologies and inexpensive commercially available resources. For instance, by using a high-throughput microfluidics approach, oil-water interfaces can be controlled in the presence of a surfactant to generate nanoliter-scale culture systems for encapsulating bacteria in oil- or water-based materials and thus to perform quick biological assays. In a few examples, monodisperse single, ${ }^{7}$ or double and tripled emulsions were generated using fluorinated and silicon oil droplets to study microbial communities and biofilm formations. ${ }^{8}$ Similarly, agarose microdroplets encapsulating bacteria were successfully created using a flow-focusing microfluidic approach. The resulting microcolonies of E. coli $(\sim 1-50 \mathrm{pL})$ were evaluated in a drug assay by means of flowcytometry and fluorescence-activated cell sorting to confirm the $\beta$-subunit of RNA polymerase as the target of the antibiotic rifampicin. ${ }^{9}$ Although the low viscosity of the oils favors the high-throughput generation of these culture systems, their fragility raises some concern for a sustainable design that would survive the high-shear or high-pressure requirements of environmental conditions found in soil or sea water. Even if the mechanical properties of the oil-based systems could be reinforced by various 
materials such as agarose, it is still critical to incorporate materials that are not degraded by the microorganisms..$^{10}$

To overcome this challenge, we previously designed a nanoliter culture system (the nanocultures) with Sylgard® 184 silicon elastomer (Dow Corning Corporation), a PDMS-based polymer. The mixture was supplemented with silicon oil to lower the viscosity of the PDMS and facilitate high-throughput microencapsulation of bacteria and fungi using a flow-focusing microfluidic device. ${ }^{11}$ Because Sylgard ${ } 184$ crosslinks and forms a semi-permeable membrane, the cells were confined within a mechanically robust housing, allowing the diffusion of gases and small molecules in and out of the sessile drops. Moreover, because chemical communication across the membrane occurred, the effects of the physical and chemical interactions on the growth dynamics of the sessile communities could be decoupled and microbial pathophysiology investigated. ${ }^{11,12}$ Sylgard® 184 was chosen for its hydrophobicity, low cost, easy processability, optical transparency, biocompatibility, thermal stability, and most importantly, its high permeability to gas, small and low-molecular weight hydrophobic molecules. Owing to these properties, water-in-oil-in-water (w/o/w) emulsions can successfully be generated to encapsulate microbes, and directly interrogate the nanocultures' responses to various molecular probes (e.g., antibiotics, sugars and dyes) to capture community dynamics as desired. Nonetheless, the undefined permeability of Sylgard ${ }^{\circledR} 184$ for most molecules, and its low chemical compatibility poses challenges and limits its applicability. ${ }^{13}$ Because of its proprietary blend, the precise chemical composition of Sylgard ${ }^{\circledR} 184$ is unknown, which affects one's ability to functionalize the polymer. An important functionality for our biological applications consists in controlling the polymer network of the PDMS, which would allow for diffusion of desired molecules through the shell of the nanocultures. Having the ability to fine-tune the transport as well as the mechanical properties of the PDMS-based nanocultures would enable us to design a high-throughput micro-isolation culture system capable of overcoming the barriers posed by conventional methods. 
In this study, we have experimentally determined the composition of a novel PDMS membrane with desired transport and mechanical properties for the microencapsulation of bacterial cultures. The PDMS membranes were developed through hydrosilylation by reacting various vinyl-terminated polydimethylsiloxane and methylhydrosiloxane-dimethylsiloxane copolymers in the presence of a platinum $(\mathrm{Pt})$ catalyst. The new membranes were characterized with Fourier Transform Infrared Spectroscopy (FTIR), and their wettability was evaluated using contact angle measurements to ensure their hydrophobicity. PDMS microcapsules were generated, and their stability and mechanical robustness were determined by osmotic pressure. A condition was therefore achieved to generate welldefined microbial nanocultures with improved transport and mechanical properties. The present study lays the foundation for a novel and functional microbial culture system that will enable the cultivation of microorganisms in environmental conditions.

\section{Results and Discussion}

\subsection{Synthesis and Mechanical Characterization of the novel PDMS Membranes}

Novel PDMS membranes were prepared through screening and mixing of various vinyl-terminated polydimethylsiloxane bases with a methylhydrosiloxane-dimethylsiloxane copolymer crosslinker as shown in Figure 1. In the presence of a Pt catalyst, the mixtures of vinyl and hydride (MeHSiO) functional groups polymerize through a reaction known as hydrosilylation. Four commercially available vinyls (DMS-V21, DMS-V31, DMS-V41, and DMS-V51) were mixed to a hydride (HMS053) in the presence of $1.5 \mathrm{ppm}$ Pt catalyst, degassed for $30 \mathrm{~min}$ in a vacuum chamber, and cured at $70^{\circ} \mathrm{C}$ for $3 \mathrm{~h}$ to generate the membranes. The membranes were initially mixed at molar ratios of the functional groups of 1:1 and 1:5 (hydride:vinyl) to assess their mechanical properties (Figure 2) and select for the ones suiting our biological application. To assess the mechanical properties of the membranes, stress-strain curves were recorded at a strain rate of $0.15 \mathrm{~min}^{-1}$ using a tensile testing machine (Instron). All polymer combinations were successfully crosslinked, though some membranes did not exhibit favorable 
properties for microbial encapsulation because of being too sticky, slimy, or crosslinking during the degassing process. Out of the all combinations, three exhibited a brittle behavior, while one displayed a rubbery deformation (Figure 2). The mixtures containing vinyl DMS-V21, -V41 and -V51 resulted in membranes with stronger mechanical properties compared to the one with DMS-V31. However, the combinations with DMS-V41 and -V51 were discarded because they crosslinked during degassing and contained bubbles. This was not suitable for our application because it could affect the stability and the optical properties of the resulting microcapsules. Also, microbial encapsulation is commonly performed at room temperature $\left(21^{\circ} \mathrm{C}\right)$ and lasts between $2-3 \mathrm{~h}$. Therefore, it is critical that the successful mixtures remain uncured during the process. Finally, the viscosity of the mixtures with DMS-V41 and -V51 was very high, which would have compromised their ability to easily flow within the microfluidic device to generate double emulsions. In the past, the viscosity of Sylgard® 184 , which is $5,100 \mathrm{cP}$, was reduced by half through the addition of silicon oil to flow through the device and generate the double emulsions. However, the mixture with DMS-V41 and -V51 has a viscosity comprised between 9,000-95,000 cP, making it less ideal for the encapsulation process (Table 2). Although the membranes were mechanically strong, with an elastic modulus varying between $38-60 \mathrm{KPa}$, they were not retained for our application (Figure 2).

Similarly, the membranes with DMS-V31 were discarded because they resulted in major deformation under tensile stress as low as $0.05 \mathrm{MPa}$, as shown in Figure 2. Although initially less viscous (viscosity $<1,000 \mathrm{cP}$, Table 2), the mixture with DMS-V31 and HMS053 generated very soft membranes that appeared too slimy and rubbery. The rubbery property did not completely disappear even when the mixing ratio of hydride and vinyl was modified.

The most successful mixture was attained with DMS-V21, the vinyl-terminated PDMS base with the lowest molecular weight $\left(6000 \mathrm{~g} \cdot \mathrm{mol}^{-1}\right)$ of all the ones tested. The mixture had an initial viscosity of $\sim 250 \mathrm{cP}$, and at a mixing molar ratio of 1:1 generated membranes exhibiting $\sim 100 \%$ strain deformation before brittle fracture under a tensile stress of $0.3 \mathrm{MPa}$. However, the mechanical strength and 
deformation of the materials was significantly reduced with the addition of more crosslinker. At a 1:5 hydride to vinyl ratio, the membrane underwent a more rapid brittle fracture corresponding to maximum deformation of $\sim 50 \%$, for a total tensile stress of $0.1 \mathrm{MPa}$. This represents an elastic modulus of $28.8 \pm 2.1$ $\mathrm{KPa}$ in contrast to $48.1 \pm 3.3 \mathrm{KPa}$ for the equimolar mixture. This novel combination of PDMS membranes with HMS053 and DMS-V21 exhibits a mechanical behavior quite different from Sylgard® 184. Silgard® 184 membranes are stronger and exhibit a rubberic behavior with an elastic modulus comprised between 1-2 MPa. ${ }^{14}$ The lower mechanical strength achieved with PDMS membranes, and more importantly, their brittle nature, provides enough mechanical stability to sustain a microbial culture in multiple environmental conditions, and also facilitates cell release from the microcapsules through mechanical stress lower than $1 \mathrm{MPa}$, which appears to be challenging with Sylgard® 184 microcapsules.

\subsection{Hydrosilylation Reaction and Hydrophobicity of PDMS Membranes}

The novel PDMS membranes present interesting mechanical properties in addition to their defined composition, which make them ideal for some biological applications. To further assess their applicability for microbial microencapsulation, the membranes were characterized through FTIR, spincoating, and contact angle measurements. Initially, PDMS membranes made at an equal mixing ratio of functional groups and with $1.5 \mathrm{ppm}$ Pt catalyst were synthesized. The HMS053 hydride used in the synthesis of the membranes contained on average $5 \%$ of MeHSiO functional group, corresponding to a molecular weight of $1000-1250$ g.mol ${ }^{-1}$. The weight percentage of the vinyl group in DMS-V21 is $1 \%$, for a total molecular weight of $6000 \mathrm{~g} \cdot \mathrm{mol}^{-1}$. Because the membranes crosslink through a hydrosilylation reaction (Figure 1), we anticipated that the FTIR analysis of the PDMS membranes in reference to the base (HMS053) and the crosslinker (DMS-V21) would result in minimal changes in the intensity of $-\mathrm{Si}-\mathrm{H}$ (Hydride) or $-\mathrm{Si}-\mathrm{CH}=\mathrm{CH}_{2}$ (vinyl) bands, if detected. The $-\mathrm{Si}-\mathrm{H}$ bands are commonly recorded at frequencies of $2280-2080$ and $950-800 \mathrm{~cm}^{-1}$, while the $-\mathrm{Si}-\mathrm{CH}=\mathrm{CH}_{2}$ bands are identified at

1600, 1418, 1010, $960 \mathrm{~cm}^{-1} .{ }^{15,16}$ The disilylethylene (-Si- $\left.\mathrm{CH}_{2}-\mathrm{CH}_{2}-\mathrm{Si}-\right)$ group, resulting from the 
hydrosilylation reaction, forms a sharp band at frequencies comprised between $1180-1120 \mathrm{~cm}^{-1}$, but only if the group is not incorporated in a strained ring. In our conditions, the FTIR spectrum revealed the presence of the vinyl bands at a frequency of $910 \mathrm{~cm}^{-1}$ in the pure DMS-V21 but not in HMS053 samples, as shown in Figure 3a. This was expected since the hydride did not contain any $-\mathrm{Si}-\mathrm{CH}=\mathrm{CH}_{2}$. The vinyl band significantly diminished in the cross-linked PDMS membranes. This decrease corresponds to the saturation of the double bond during the crosslinking process with the hydride, resulting in a polymer network with single $\mathrm{C}-\mathrm{C}$ bonds in place of the vinyl double bonds. However, the inability to identify the $\mathrm{Si}-\mathrm{H}$ and $-\mathrm{Si}-\mathrm{CH}_{2}-\mathrm{CH}_{2}-\mathrm{Si}-$ bands could be attributed to the low concentration of this functional group in the samples.

Following FTIR characterization, the mixtures were used to generate thin polymer membranes via spin coating, as shown in Figure 3b. Microbial encapsulation requires the containment of an aqueous suspension in thin polymer membranes, therefore it was essential to prove that a thin membrane could be achieve with this low-viscosity mixture. Hence, the PDMS mixture was spun at 300 RPM and 800 RPM to create membranes with thicknesses varying between 21 and $140 \mu \mathrm{m}$. While the generation of thin films would be appropriate to study the transport properties of the membranes, in our case it was also relevant to assess the uniformity of the curing process as well as the optical property of the thin membranes required for the shell of the nanocultures (Figure 3b).

Moreover, the hydrophobicity of the PDMS membranes was characterized via contact angle measurements with $2 \mathrm{~nL}$ water and ethanol droplets. The solvents were used because they are involved in the cleaning of the microfluidic device and the generation of the nanocultures. The contact angle is determined by analyzing the shape of the droplet in reference to the 3-point interface between solid membrane, liquid droplet and ambient air. Hydrophilicity is indicated by a contact angle smaller than $90^{\circ}$, whereas hydrophobicity is indicated by a contact angle larger than $90^{\circ}$. As shown in Figure $3 \mathbf{c}$, the PDMS membranes are hydrophobic with a contact angle of $109.0 \pm 0.1^{\circ}$, which is consistent with measurements made on other PDMS membranes, such as Sylgard ${ }^{\circledR} 184 \cdot{ }^{17,18}$ In regard to the PDMS 
membranes, the change in the mixing ratio of the hydride and vinyl from 1:2 to $2: 1$ did very minimally affect their hydrophobicity by an order of $0.2^{\circ}$ (Figure 3c, $p<0.0001$ ), suggesting that polymer samples with hydride to vinyl mixing ratios ranging between 1:2 to $2: 1$ will be ideal for generating double emulsions for microbial encapsulation because of their hydrophobicity. ${ }^{19}$

The ethanol contact angle was measured to determine if the membrane appeared to have a strong affinity for ethanol. As seen in the contact angle value of $36.6 \pm 1.4^{\circ}$ for the membrane generated at equal molar ratio. However, the ethanol contact angle further decreased with change in the hydride/vinyl ratio, illustrating the superwetting properties of ethanol to the PDMS membranes, more especially at a higher vinyl ratio $\left(\mathrm{ECA}=18.2 \pm 0.2^{\circ}\right.$; Figure 3c, $\left.p<0.0001\right)$. The low ethanol contact angle of the PDMS membrane implies a high surface energy and interfacial tension, and consequently indicates a high miscibility between the polymer mixture and ethanol before crosslinking. ${ }^{20}$ It is therefore not surprising that ethanol is ideal to clean the uncured polymer from microfluidic devices and completely flush the PDMS crosslink to avoid device clogging. Both the water and the ethanol contact angle measurements confirm that the PDMS membrane was ideal for water phase separation, and therefore for generation $\mathrm{w} / \mathrm{o} / \mathrm{w}$ emulsions.

\subsection{Generation and Mechanical Stability of the novel PDMS-based Microcapsules}

We hypothesized that the novel PDMS mixtures could be used to generate stable w/o/w doubleemulsions and nanocultures because of their crosslinking ability, mechanical properties, and hydrophobicity. To verify this hypothesis, the crosslinker (HMS053) and the base (DMS-V21) were optimally mixed at a 0.6:1 ratio of the functional groups and supplemented with $2 \mathrm{ppm}$ Pt catalyst, degassed in a vacuum for $30 \mathrm{~min}$, and introduced in the glass capillary microfluidic device as the middle phase. Monodisperse double-emulsions were successfully generated with a core of Milli-Q water and an outer phase of 5\% PVA solution in water (Figure 4a). However, the w/o/w double emulsions did not 
remain stable following their incubation at $37^{\circ} \mathrm{C}$ in the aqueous environment. Instead of curing into microcapsules with water cores and PDMS shells (Figure 4b), more than 70\% of the double emulsions burst within $8 \mathrm{~h}$ after their generation and converted into single emulsions, suggesting a lack of stability, as shown in Figure 4c-e (See Supporting Video 1).

An optimization test was performed to determine an adequate polymer composition for generating stable double-emulsions. First, PDMS-based double-emulsions generated with a hydride-vinyl mixture at a 0.6:1 ratio and $1.5 \mathrm{ppm}$ Pt catalyst and monitored using a light microscope (ZEISS Axio Imager 2, Carl Zeiss). In this condition, the capsules burst by $100 \%$ within 2 h, as shown in Figure 4 f. Because the membranes generated for the FTIR and contact angle characterizations were all synthesized at $70^{\circ} \mathrm{C}$, in contrast to the emulsions that were incubated at $37^{\circ} \mathrm{C}$, we anticipated that raising the incubation temperature would accelerate the hydrosilylation reaction kinetic and result in more stable capsules. Thus, after their generation, the double emulsions were briefly incubated at $70^{\circ} \mathrm{C}$ for $5 \mathrm{~min}$ before evaluating their stability at $37^{\circ} \mathrm{C}$ for a period of $15 \mathrm{~h}$, using a light microscope with a heating stage (Carl Zeiss, Inc.). The $5 \mathrm{~min}$ incubation was set as the limit for heat-pretreatment because many bacteria such as $P$. aeruginosa can survive a brief exposure to such temperature. ${ }^{21}$ As shown in Figure $\mathbf{4 f}$, the stability of the double-emulsions was improved by $15 \%$, which was not satisfying for designing a microbial culture system. We hypothesized that increasing the Pt concentration could further accelerate the hydrosilylation reaction and improve the stability of the microcapsules. Therefore, the concentration of Pt catalyst in the hydride-vinyl mixture was doubled to $3 \mathrm{ppm}$ to generate monodisperse doubleemulsions, and the number of capsules bursting within $15 \mathrm{~h}$ of their generation was quantified and plotted as a function of time. As shown in Figure 4f, the stability of the microcapsules was then improved by $\sim 65 \%$, compared to the condition with $1.5 \mathrm{ppm}$ Pt. Unfortunately, above a concentration of $3 \mathrm{ppm}$ Pt catalyst, we determined that the polymers crosslinked more rapidly, and during the encapsulation process. Therefore, the capsules generated with $3 \mathrm{ppm} \mathrm{Pt}$ catalyst were pre-treated at $70^{\circ} \mathrm{C}$ for $5 \mathrm{~min}$ to enhance their stability, which led to a survival rate of $100 \%$ (Figure $\mathbf{4 f}$ ). Using this 
combination of hydride, vinyl, Pt concentration, and heat pre-treatment, stable microcapsules were generated and functionalized with fluorescence dye such as Nile Red (Sigma Aldrich), as shown in Figure 4g. This condition for microcapsule generation was utilized to developed the microbial nanoculture system.

\subsection{Microbial Growth Dynamics in PDMS-based Nanocultures.}

We determined a condition for generating stable PDMS microcapsules, which requires heat pretreatment to initiate the curing of the polymeric shells of the capsules. To determine if this condition is conducive for developing a novel culture system, $P$. aeruginosa PAO1 cells were grown overnight in a UFTYE medium and encapsulated in a PDMS mixture containing 3 ppm Pt catalyst. The nanocultures were generated with a suspension of PAO1 cells in UFTYE media as the inner phase of the flowfocusing microfluidics device. The outer phase remained a 5\% PVA solution in Milli-Q water. The nanocultures were seeded at an average density of $\sim 5$ cells per nanoculture, collected in $154 \mathrm{mM}$ $(0.85 \%) \mathrm{NaCl}$ solution and pretreated at $70^{\circ} \mathrm{C}$ for $5 \mathrm{~min}$ before their incubation at $37^{\circ} \mathrm{C}$ overnight on the heated stage of the Zeiss microscope. Then, the growth dynamics of the PAO1 cells were monitored every $15 \mathrm{sec}$ for $20 \mathrm{~h}$ through time-lapse imaging. As shown in Figure 5, the PAO1 nanoculture became more confluent over time (See Supporting Video 2). Microbial dynamics in the nanocultures corresponded to the various growth stages of PAO1 cells, including a lag-phase $(0-7 \mathrm{~h})$, an exponential phase $(\sim 7-13 \mathrm{~h})$, and a stationary phase after $13 \mathrm{~h}$ of incubation. Interestingly, the growth dynamics of the cells correlated with a change in the size of the capsule. A minimal size change was observed during the first $7 \mathrm{~h}$, but became more significant when the cells reached exponential growth, suggesting that microbial growth in the nanoculture is highly associated with the transport properties of the microcapsules. We projected that microbial growth in a UFTYE medium would lead to the generation of waste products and chemical species within the capsules, which would in turn result in 
molecular fluxes due to a concentration gradient across the nanoculture shell. On the other hand, PVA, and sodium and chloride ions are present in the collection medium, and do not partition into the PDMS membrane, owing to their charges and high molecular weights. The $154 \mathrm{mM} \mathrm{NaCl}$ solution of the collection medium by itself would impose an osmotic pressure of $0.77 \mathrm{MPa}$ to each microcapsule, which would induce water flux across the membranes at a rate of $5.2510^{-3} \mu \mathrm{L} / \mathrm{h}$ (Figure 5). Nonetheless, the nanocultures maintained their stability during the incubation of PAO1 cells, indicating that the encapsulation condition was optimal to sustain microbial growth in the newly developed polymer.

\subsection{Transport Properties of the PDMS-based Nanocultures}

The change in the size of the nanocultures suggested that mass and momentum transfers occurred as a result of microbial growth dynamics in the nanocultures. We hypothesized that the transport properties of the nanocultures would highly depend on the degree of polymerization of their shells and the porosity of the polymer network. By conducting the osmotic annealing experiment, we aimed to qualitatively assess the degree of crosslinking and the transport properties of the microcapsules. Microcapsules containing Milli-Q water as the core and PDMS (H:V molar ratio of 0.6:1, with 3 ppm Pt catalyst) as the shell were generated, and immediately pre-treated at $70^{\circ} \mathrm{C}$ for $0 \mathrm{~min}, 5 \mathrm{~min}, 2 \mathrm{~h}$ and $4 \mathrm{~h}$ before osmotic annealing. The microcapsules were secured in a MatTek dish (MatTek) and exposed to a $10 \% \mathrm{NaCl}$ (or $1,725 \mathrm{mM} \mathrm{NaCl}$ ) solution to record the osmostically-induced size change every 10 sec for $5 \mathrm{~h}$ using a light microscope (Axio Imager 2, Carl Zeiss). The osmotic pressure applied to the microcapsules corresponded to $8.5 \mathrm{MPa}$, and exerted a hydrostatic force to drive water out and cause the capsules to shrink in size. Our results demonstrated that the degree of curing of the microcapsules did affect the flux of water out of the microcapsules (Figure 6a, Panel 1; See Supporting Videos 3). The osmotic annealing caused water to osmose and convert the untreated microcapsules into single PDMS emulsions (Figure 6b). However, the osmosis induced shrinkage in the microcapsules that were 
pre-treated at $70{ }^{\circ} \mathrm{C}$ for 5 min more rapidly (Figure 6a, Panel 2; See Supporting Video 4), which was also confirmed by tracking the radial velocity of the shrinking. As shown in Figure 6c, the untreated microcapsules decreased as a rate of $18.19 \mu \mathrm{m} / \mathrm{h}$, in contrast to those pre-treated at $70{ }^{\circ} \mathrm{C}$ for $5 \mathrm{~min}$, which shrank more rapidly at a constant velocity of $27.5 \mu \mathrm{m} / \mathrm{h}$ (Figure $\mathbf{6 d}$ ). In the latter condition, the microcapsules presented a cured and semi-solid inner-interface after $3 \mathrm{~h}$ of osmotic annealing, indicating that crosslinking was initiated and contributed to the mechanical stability of the microcapsules, in agreement with the findings reported in Figure $4 \mathbf{f}$.

Furthermore, the microcapsules were pre-treated at $70^{\circ} \mathrm{C}$ for $2 \mathrm{~h}$ and $4 \mathrm{~h}$ to accelerate the hydrosilylation kinetic and corroborate the improved mechanical and transport properties through osmotic annealing. As shown in Panels 3 and 4 of Figure 6a, the microcapsules buckled and severely deformed immediately when osmosis occurred, indicating that they were fully cured. For those microcapsules exposed to heat pre-treatment for 2 hours or longer, water fully osmosed out within $3 \mathrm{hr}$ at the osmotic pressure of $8.76 \mathrm{MPa}$ (Figure 6, Panel 3\&4; See Supporting Videos 5\&6). The fact that the membranes began to buckle, folding into themselves instead of breaking, indicate that they are mechanically strong enough to sustain growth in many environmental conditions.

In addition, we aimed to verify that the PDMS nanocultures developed for microbial encapsulation and presented in Figure 5 were fully crosslinked after incubation at $37^{\circ} \mathrm{C}$ for $24 \mathrm{~h}$. Therefore, the microcapsules generated with hydride and vinyl mixed at a ratio of $0.6: 1$ in the presence of $3 \mathrm{ppm} \mathrm{Pt}$ catalyst were incubated at $37^{\circ} \mathrm{C}$ overnight following heat pre-treatment at $70{ }^{\circ} \mathrm{C}$ for $5 \mathrm{~min}$. The capsules were then exposed to $1,725 \mathrm{mM} \mathrm{NaCl}(10 \% \mathrm{NaCl})$ solution to assess if they shrank into single emulsions (Figure 6a, Panel 2) or buckled (Figure 6a, Panel 3). Our data showed that, in the conditions used for the PDMS nanocultures, $95 \%$ of the microcapsules were fully cured after a $24 \mathrm{~h}$ incubation at $37^{\circ} \mathrm{C}$ (See Supporting Video 7). From these results, it is evident that the brief heat pre-treatment at $70^{\circ} \mathrm{C}$ for $5 \mathrm{~min}$ triggered the hydrosilylation reaction. However, the polymerization of the microcapsules continued over the course of incubation at $37^{\circ} \mathrm{C}$ until full crosslinking occurred. Although shrinking of the PAO1 
nanocultures occurred over the $20 \mathrm{~h}$ of incubation (Figure 5), we expected osmotically-induced buckling of the nanoculture system could also occur in other nanoculture systems, because a good proportion of the microcapsules cured fully within $24 \mathrm{~h}$ (See Supporting Video 2). This hypothesis was verified when nanocultures with RFP-labelled Escherichia coli cells were generated as described above. As shown in Figure 7a, the nanocultures did experience buckling under osmotic pressure, indicating that they were fully crosslinked. The buckling of the shell suggests that water flux out of the nanocultures occurred at a time when the nanocultures were already crosslinked. These results indicate that microbial dynamics might lead to undesired transport processes, which could affect not only the mechanical integrity of the nanocultures, but also their cell densities. Because the UFTYE medium does not contain $\mathrm{NaCl}$, it was expected that a flux of water against the $\mathrm{NaCl}$ concentration gradient would occur. This could be prevented if the culture medium was present in either side of the polymeric shell or if the nanocultures were incubated in an isotonic condition. To test how such culture conditions would affect microbial growth, nanocultures containing a suspension of RFP-labelled E. coli cells in UFTYE were collected in the MatTek dishes containing the UFTYE medium instead of a $154 \mathrm{mM} \mathrm{NaCl}$ solution. In contrast to Figure 7a, the nanocultures that were resuspended in the UFTYE medium did not experienced any buckling (Figure 7b). All the capsules maintained their integrity by remaining spherical. Additionally, a more confluent microbial growth was achieved in the isotonic condition. To compare cell confluence in both conditions, fluorescent images were acquired using a Zeiss microscope. Z-stack images were recorded to measure cell confluence in the nanocultures (See Supporting Videos 8\&9). The results reveal that the fluorescence intensity of the nanocultures collected in the UFTYE medium was twice that recorded with those resuspended in a $154 \mathrm{mM} \mathrm{NaCl}$ solution (Figure 7c, $p<0.0001$ ). A fluorescence intensity of 31,938 a.u. was measured with the cells cultured in the isotonic condition, vs. 14,263 a.u. for the E. coli RFP nanocultures grown in the hypertonic condition (with $154 \mathrm{mM} \mathrm{NaCl}$ ).

These results are intriguing, as they indicate that there are many transport processes associated with the design of these novel microbial culture systems. To better manipulate microbial dynamics in 
nanocultures, a system with improved transport and mechanical properties is essential. The ability to determine all the metabolites and chemical species arising from the microbial dynamics is as important as is a good understanding of the chemical compositions of the polymer used to generate the shells, of the cores, and of the collection medium as well. This will help achieve a more porous polymer network, which facilitate the flux desired molecules based on their size and chemical functionality. It is evident that the degree of polymerization of the PDMS membranes is directly linked to the crosslinking ability of the reacting functional groups, and their transport and mechanical properties. Hence, by determining the ratio of the concentration of the functional groups of each reagent, we anticipate being able to tune the degree of polymerization, the amount of free space within the network, and the molecular selectivity of the membranes. However, the design of the polymeric shell achieved in this study provides a new platform for developing a more functional system, which could be chemically modified for various applications. For instance, the membrane can be functionalized with N,N-Dimethylallylamine to act as a spacer and compete against vinyl-terminated PDMS in order to achieve a more permeable PDMS membrane. Such functionalization of the PDMS membrane is part of our ongoing work, and will help generate new culture systems to grow, sequester, and deliver microbial cultures into various microenvironments, on demand.

\section{Conclusion and Perspectives}

This study presents a novel microbial nanoculture system designed with a defined composition of PDMS-based hydride and vinyl. With an initial low viscosity, the polymer mixture crosslinked into a membrane with stable mechanical properties to house microorganisms in the nanocultures. The characterization of the transport properties of the materials provided new evidence that a more tunable microbial culture system can be achieved by controlling the mixing ratio of the PDMS-based hydride and vinyl polymers, as well as the cell microenvironments. The results of this research provide a unique platform to test how the diffusion antibiotics, dyes, signaling and other bioactive molecules can be used 
to interrogate microbial dynamics and diversity. With recent advances in the understanding of the human microbiome and its implication for health, such innovative culture methods become essential for improving microbial culturability and manipulating microbial dynamics. Thus, the present study sets the stage for deliverable microsystem platforms that are also biocompatible. Further functionalizations of the novel PDMS nanoculture system will be significant to the design of swallowable devices for the localized delivery of microbial-based therapies.

\section{Experimental Section}

Polymer Synthesis: Vinyl-terminated PDMS including DMS-V21, DMS-V31, DMS-V41, and DMSV51, and Methylhydrosiloxane-Dimethylsiloxane Copolymer and Trimethylsiloxane Terminated (HMS053) were purchased from Gelest, Inc. The polymers were chosen based on varying molecular weight and viscosity (Table 1). The hydromethylsiloxane copolymer and the vinyls were mixed at molar ratios of $0.6: 1,1: 1$ or 1:5 (Hydride: Vinyl) to a total mass of $4 \mathrm{~g}$. The product mixture was added to a PTFE evaporating dish (Fisher) along with a platinum (Pt)-divinyltetramethylsiloxane complex (2\% Pt in xylenes) catalyst to initiate the hydrosilylation reaction. The mixture was stirred for 5 min and degassed under vacuum for 15-30 min to remove any air bubbles. The dish was then placed in an oven at $70^{\circ} \mathrm{C}$ to cure for approximately $3 \mathrm{~h}$ to generate the novel polydimethylsiloxane membranes.

Mechanical testing: The tensile strength of the PDMS membranes was evaluated on a tensile testing machine (Instron). The membranes were molded into samples with uniform length, width, and thickness $(40 \mathrm{~mm} \times 5 \mathrm{~mm} \times 5 \mathrm{~mm})$, and mounted in the pneumatic clamp of the Instron system at a $20 \mathrm{~mm}$ gauge length and a $5 \mathrm{~mm}$ width. The membrane was pulled apart at a rate of $3 \mathrm{~mm} / \mathrm{min}$, and the displacement was recorded until failure of the samples. The data obtained from the test included force $(\mathrm{N})$ and extension (mm) in the form of an x-y plot. Each polymer mixture was tested at least in triplicate. 
FTIR Analysis. Novel PDMS membranes were prepared with DMS-V21 and HMS053 mixed at 1:1 molar ratio along with $1.5 \mathrm{ppm}$ Pt. The samples were cured at $70^{\circ} \mathrm{C}$ for $3 \mathrm{~h}$ and placed over the diamond crystal of the FTIR unit (Nicolet ${ }^{\mathrm{TM}}$ iS50 FTIR spectrometer, Thermo Fisher). Finally, the samples were analyzed using the Attenuated Total Reflectance (ATR) method over an accumulative 32 scans in the range of $4000 \mathrm{~cm}-1$ to $400 \mathrm{~cm}^{-1}$. Similarly, the hydride (HMS053) and the vinyl (DMS-V21) were placed over the diamond crystal holder to record the FTIR spectra of the control samples.

Spin Coating. A silicon wafer was introduced in a 5.0\% PVA solution, then dried in an oven at $95^{\circ} \mathrm{C}$ to allow the formation of a uniform PVA layer on the wafer. To generate thin PDMS membranes, mixtures prepared with DMS-V21 and HMS053 at a 1:1 molar ratio and supplemented with $1.5 \mathrm{ppm}$ Pt were degassed as described above, poured on the PVA-coated silicon wafer, and spun at rotations ranging from 400 RPM - 800 RPM for $30 \mathrm{sec}$ using the Laurell WS-650Mz-23NPPB Spin Processor (Laurell). Finally, the membranes were cured at $95^{\circ} \mathrm{C}$ overnight, measured using a Profilometer surface profiler (KLA-Tencor Alpha-Step IQ), and imaged using a camera.

Contact Angle Measurement. PDMS mixtures prepared with DMS-V21 and HMS053 at molar ratios varying between 1:2 and 2:1 were supplemented with $1.5 \mathrm{ppm}$ Pt. The mixtures were degassed in a vacuum as described above, then cured at $95^{\circ} \mathrm{C}$ overnight. To characterize the hydrophobicity of the membranes, water contact angle was measured using the optical tensiometer Attension Theta (Biolin Scientific) with a $2 \mathrm{~nL}$ droplet of water or ethanol. 
Microorganisms and growth conditions. The protocol described by Niepa et al. ${ }^{11}$ was followed with slight modifications. P. aeruginosa PAO1 and E. coli RP437motB580/pRSH103 ${ }^{22}$ were utilized to generate the nanocultures. The cells were cultured in a buffered low-molecular weight medium referred to as an ultrafiltered tryptone-yeast extract (UFTYE) broth. The UFTYE containing $2.5 \%$ tryptone and $1.5 \%$ yeast extract had a molecular-weight cut-off of $10 \mathrm{kDa}$ (Millipore). ${ }^{12} \mathrm{The} \mathrm{pH}$ of the medium was adjusted to 7 to grow the cells. Briefly, $25 \mathrm{~mL}$ of UFTYE were introduced in a flask to culture the cells overnight. Then, $50 \mu \mathrm{L}$ aliquots were introduced in $5 \mathrm{~mL}$ of UFTYE to constitute the inner phase of the microfluidics, as described above. The middle phase was encapsulated in PDMS mixtures to generate nanocultures, which were incubated at $37^{\circ} \mathrm{C}$.

Fabrication of microfluidic devices. The protocol described by Niepa et al. ${ }^{11}$ was followed with slight modifications. We made use of a glass-capillary microfluidic device with hydrodynamic flow-focusing and coflowing geometry to generate the w/o/w PDMS microcapsules. Briefly, two circular capillary tubes with inner and outer diameters of $0.58 \mathrm{~mm}$ and $1.03 \mathrm{~mm}$ (World Precision Instrument) were tapered to the desired diameters using a Sutter P-1000 Horizontal Micropipette Puller (Sutter Instrument) and a MF-900 microforge (Narishige). The inner diameters of the tapered tubes for the injection of bacteria phase and the collection of capsules were $40 \mu \mathrm{m}$ and $200 \mu \mathrm{m}$, respectively. The outside of the glass capillary tube for inner fluid was hydrophobically functionalized with $1 \%$ octadecyltrichlorosilane (OTS, Sigma-Aldrich) in toluene. This chemical treatment enhances the wettability of the PDMS mixture outside the capillary tube, and facilitates the formation of capsules. The two tapered capillary tubes were inserted into a square capillary with an inner dimension of 1.05 $\mathrm{mm}$, and separated by a distance of $120 \mu \mathrm{m}$. Subsequently, a transparent epoxy was used to seal the tubes where required. 
Generation of the nanocultures. The microfluidic device was mounted on an inverted optical microscope (Eclipse TE300, NIKON). Then, the three fluid phases were delivered to the microfluidic device through polyethylene tubing (Scientific Commodities) that were attached to syringes (SGE) that were driven by positive displacement syringe pumps (Harvard Apparatus, Standard PHD ULTRA ${ }^{\mathrm{TM}}$ CP). The drop formation was monitored with a Phantom VEO 710L high-speed camera (Vision Research) attached to the inverted microscope. The inner aqueous phase consists of bacteria suspended in the culture medium; the middle phase consists of PDMS mixtures with DMS-V21 and HMS053 at a molar of 0.6:1, supplemented with $1.5-3 \mathrm{ppm}$ Pt (Gelest). The outside phase comprises $5 \mathrm{wt} \%$ poly(vinyl alcohol) aqueous solution (PVA, 87-89\% hydrolyzed, average $\mathrm{M}_{\mathrm{w}}=13,000-23,000$, Aldrich). The stability of the microcapsules was evaluated for $15 \mathrm{~h}$ using a Zeiss Axio Imager 2 light microscope (Carl Zeiss,). The microcapsules made with a water (Milli-Q) core were collected in Milli-Q water and secured in a MatTek dish to record the number of microcapsules that burst and converted into single emulsions. The number of stable double-emulsions was plotted as a function of time.

Bacterial Growth Dynamics. Bacterial growth dynamics is studied by collecting the nanocultures in a $154 \mathrm{mM} \mathrm{NaCl}$ solution or UFTYE medium contained in a MatTek dish covered with a coverslip. The nanocultures were grown at $37^{\circ} \mathrm{C}$ on a heated stage and imaged using a light or epi-fluorescence microscope with 10x or 50x objectives (Carl Zeiss Inc.). The number of bacteria and the size of the nanocultures were recorded each $15-30 \mathrm{sec}$ via time-lapse imaging for 20-24 h. Image $\mathbf{J}$ was used to process the images and analyze the cells count of the nanoculture.

Osmotic Annealing. To characterize the mechanical strength and the degree of polymerization of the microcapsules, the PDMS mixture was prepared with DMS-V21 and HMS053 at molar ratio 0.6:1, and supplemented with $3 \mathrm{ppm}$ Pt. The microcapsules were generated and pre-treated with heat at $70^{\circ} \mathrm{C}$ for 0 
min, $5 \mathrm{~min}, 2 \mathrm{~h}$ and $4 \mathrm{~h}$ and introduced into a $10 \% \mathrm{NaCl}$ solution to record the osmotically-induced deformation of the capsules as a function of time. The microcapsules were secured in the MatTek dish to prevent motion during the osmotic annealing. The size of the capsules was recorded every $15 \mathrm{sec}$ for $7 \mathrm{~h}$ using a light microscope (Carl Zeiss, Inc). The images were processed using Image $\mathbf{J}$ to determine the evolution of the radius of the microcapsules and plot it as a function of time.

Statistical Analysis. JMP14.1 Software (SAS Institute, NC) was used to conduct one-way ANOVA and the t-test, and establish the significance of the testing conditions. Differences with $p<0.05$ were considered statistically significant. The following notations "ns", *, **, ***, and **** describe the statistical difference with $p$ values corresponding to $p>0.05, p<0.05, p<0.01, p<0.001$, and $p<$ 0.0001 , respectively.

\section{Data Availability}

The data that support the findings of this study are available from the corresponding author upon reasonable request.

\section{Acknowledgements}

We are grateful to Prof. David Vorp and Dr. Timothy Chung (University of Pittsburgh) for providing and assisting with the use of the Tensile testing machine. We also thank Prof. Dacheng Ren (Syracuse University) for providing the E. coli strains. This work was completed in part through generous startup funds provided by the University of Pittsburgh.

\section{Competing Interests}

The authors declare that there are no competing interests 


\section{Author Contribution}

H.U., E.J.B., and T.H.R.N. conceived the experiments, H.U., S-L.D., N.H.M., J.T.N., A.B., and R.S. conducted the experiments, H.U. and T.H.R.N. analyzed the results, E.J.B., and T.H.R.N. supervised the work. H.U., S-L.D., and T.H.R.N wrote the manuscript. All authors have reviewed and given approval to the final version of the manuscript. 


\section{References}

1 Stewart, E. J. Growing Unculturable Bacteria. Journal of Bacteriology 194, 4151, doi:10.1128/JB.00345-12 (2012).

2 Bernard, G., Pathmanathan, J. S., Lannes, R., Lopez, P. \& Bapteste, E. Microbial Dark Matter Investigations: How Microbial Studies Transform Biological Knowledge and Empirically Sketch a Logic of Scientific Discovery. Genome Biology and Evolution 10, 707-715, doi:10.1093/gbe/evy031 (2018).

3 Kato, S. et al. Isolation of Previously Uncultured Slow-Growing Bacteria by Using a Simple Modification in the Preparation of Agar Media. Applied and Environmental Microbiology 84, doi:10.1128/aem.00807-18 (2018).

4 Chaudhary, D. K., Khulan, A. \& Kim, J. Development of a novel cultivation technique for uncultured soil bacteria. Scientific Reports 9, 6666, doi:10.1038/s41598-019-43182-x (2019).

5 Nichols, D. et al. Use of ichip for high-throughput in situ cultivation of "uncultivable" microbial species. Appl. Environ. Microbiol. 76, 2445-2450 (2010).

6 Berdy, B., Spoering, A. L., Ling, L. L. \& Epstein, S. S. In situ cultivation of previously uncultivable microorganisms using the ichip. Nature protocols 12, 2232 (2017).

7 Holtze, C. et al. Biocompatible surfactants for water-in-fluorocarbon emulsions. Lab on a Chip 8, 1632-1639 (2008).

8 Chang, C. B., Wilking, J. N., Kim, S. H., Shum, H. C. \& Weitz, D. A. Monodisperse emulsion drop microenvironments for bacterial biofilm growth. Small 11, 3954-3961 (2015).

9 Eun, Y.-J., Utada, A. S., Copeland, M. F., Takeuchi, S. \& Weibel, D. B. Encapsulating bacteria in agarose microparticles using microfluidics for high-throughput cell analysis and isolation. ACS chemical biology 6, 260-266 (2011).

10 Chi, W.-J., Chang, Y.-K. \& Hong, S.-K. Agar degradation by microorganisms and agardegrading enzymes. Applied microbiology and biotechnology 94, 917-930 (2012). 
11 Niepa, T. H. et al. Microbial nanoculture as an artificial microniche. Scientific reports 6, 30578 (2016).

12 Kim, D. et al. Candida albicans stimulates Streptococcus mutans microcolony development via cross-kingdom biofilm-derived metabolites. Scientific Reports 7, 1-14 (2017).

13 Naeimi, M., Karkhaneh, A., Barzin, J., Khorasani, M. T. \& Ghaffarieh, A. Novel PDMS- based membranes: Sodium chloride and glucose permeability. Journal of applied polymer science 127, 3940-3947 (2013).

14 Khanafer, K., Duprey, A., Schlicht, M. \& Berguer, R. Effects of strain rate, mixing ratio, and stress-strain definition on the mechanical behavior of the polydimethylsiloxane (PDMS) material as related to its biological applications. Biomedical microdevices 11, 503 (2009).

15 Anderson, D. \& Smith, A. L. Analysis of silicones. Wiley-Interscience, New York, 247 (1974).

16 Launer, P. \& Arkles, B. Silicon Compounds: Silanes and Silicones. Gelest, Inc, 175-178 (2013).

17 Wang, C., Fuller, T., Zhang, W. \& Wynne, K. J. Thickness dependence of ice removal stress for a polydimethylsiloxane nanocomposite: Sylgard 184. Langmuir 30, 12819-12826 (2014).

18 Zhang, H. \& Lee, N. Y. Non-silicon substrate bonding mediated by poly (dimethylsiloxane) interfacial coating. Applied Surface Science 327, 233-240 (2015).

19 Ruan, G., Feng, S.-S. \& Li, Q.-T. Effects of material hydrophobicity on physical properties of polymeric microspheres formed by double emulsion process. Journal of controlled release $\mathbf{8 4}$, $151-160(2002)$.

20 Packham, D. Work of adhesion: contact angles and contact mechanics. International journal of adhesion and adhesives 16, 121-128 (1996).

21 O'Toole, A., Ricker, E. B. \& Nuxoll, E. Thermal mitigation of Pseudomonas aeruginosa biofilms. Biofouling 31, 665-675 (2015). 
22 Gu, H., Hou, S., Yongyat, C., De Tore, S. \& Ren, D. Patterned biofilm formation reveals a mechanism for structural heterogeneity in bacterial biofilms. Langmuir 29, 11145-11153 (2013). 


\section{List of Figures}

1. Hydrosilylation Reaction. Chemical structure of Vinyl-Terminated PDMS and the MethylHydrosiloxane-Dimethylsiloxane copolymer used as the bases and the crosslinkers of the novel PDMS membranes. The membranes were synthetized through a hydrosilylation reaction between the vinyl and the hydride (MeHSiO) in the presence of a platinum catalyst....

2. Mechanical Properties of the Novel Polydimethylsiloxane Membranes. Commercially available vinyls including DMS-V21, DMS-V31, DMS-V41, and DMS-V51 and a hydride (HMS053) mixed at ratio of 1:1 and 1:5 to generate PDMS 21-53, PDMS 31-53, PDMS 41-53, and PDMS 51-53 membranes, respectively. The tensile properties of new membranes were measured at a strain rate of $0.15 \mathrm{~min}^{-1}$. The average tensile strength is presented for each mixing ratio and combination .28

3. Characterization of new PDMS Membranes. (a) FTIR spectra were collected for the crosslinker (hydride), the base (vinyls) and the PDMS membranes. Peak at $\sim 910 \mathrm{~cm}^{-1}$ refers to the vinyl carbon-carbon double bonds, which become saturated during the hydrosilylation reaction and crosslinking process. (b) Spin-coating of PDMS mixture onto silicon wafer at 300500 RPM generates transparent membranes with thicknesses ranging between 21-140 $\mu \mathrm{m}$. (c) Water and ethanol contact angles were measured for a $2 \mathrm{~nL}$ droplet on PDMS membranes made with varying functional group concentrations. .29

4. Generation of PDMS Microcapsules using a Flow-Focusing Microfluidic Device. (a) The 3 liquid phases were introduced into the device and directed to a 3-phase interface for highthroughput generation of monodisperse w/o/w double-emulsions. As illustrated, the aqueous culture medium, the PDMS mixtures, and the PVA solution constitute the 3 phases involved in the w/o/w double emulsions. (b) Mechanical instability converts double emulsions into single emulsions. Successful crosslinking of PDMS membranes lead to stable microcapsules. (c-e) Uncured PDMS capsules become unstable and burst into single emulsions. Scale bar: $50 \mu \mathrm{m}$ (f) PDMS microcapsule stability is improved with the addition of Pt catalyst and heat pretreatments. (g) Stable PDMS microcapsules are achieved by mixing the hydride and vinyl at a 0.6:1 ratio, supplementing the mixture with $3 \mathrm{ppm} \mathrm{Pt}$ catalyst and heatpretreatment at $70^{\circ} \mathrm{C}$ for 5 min. Scale bar: $150 \mu \mathrm{m}$

5. Growth Dynamics of PAO1 Cells in the Nanocultures. A grayscale rendering of the PDMS nanoculture recorded for $20 \mathrm{~h}$ displays a confluent growth of Pseudomonas aeruginosa. The exponential growth stage is achieved between 7-13 $\mathrm{h}$ and the stationary phase is attained after $13 \mathrm{~h}$ of incubation. Incubation of PAO1 nanocultures in $154 \mathrm{mM} \mathrm{NaCl}$ results in a sharp decrease of size of the microcapsules. Scale bar: $50 \mu \mathrm{m}$. 
6. Transport and Mechanical Properties of PDMS Microcapsules. The microcapsules were subjected to osmotic pressure in a $10 \% \mathrm{NaCl}$ solution for 7 hours, to determine the mechanical stability of the capsules after heat treatment. (a) Osmotic annealing induced shrinkage of uncured (Panel $1 \& 2$ ) and buckling of cured (Panel $3 \&$ \& ) PDMS microcapsules. Polymerization of PDMS membrane is initiated after heat exposure at $70^{\circ} \mathrm{C}$ for $5 \mathrm{~min}$ as seen with the solid interface in Panel 2. Scale bar: $50 \mu \mathrm{m}$ (b) Schematic illustration of the osmotically-induced shape deformation of the microcapsules. The inner $\left(\Phi_{\mathrm{i}}\right)$ and outer $\left(\Phi_{\mathrm{o}}\right)$ radii of the microcapsules change as a function of time. Radial change in the shrinking microcapsules is compared before (c) and after (d) heat pre-treatment at $70^{\circ} \mathrm{C}$ for $5 \mathrm{~min}$. The slope of the fit for the radial evolution of the inner droplet describes the radial velocity of the water flux during osmosis

7. Transport Process Associated with PDMS Nanocultures. PDMS nanocultures containing RFP-labelled E. coli suspension in UFTYE were incubated in (a) hypotonic (154 mM NaCl) solution vs. (b) isotonic (UFTYE) conditions. While incubation in the hypertonic condition leads to buckling of the crosslinked polymeric shell, the nanocultures grown in the isotonic condition maintain their mechanical integrity. (c) Fluorescent imaging demonstrates that a high cell density is achieved in the isotonic condition. Scale bar: 50

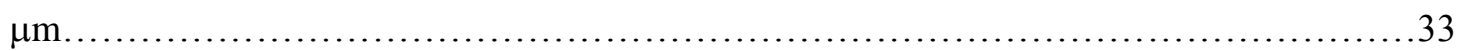

8. Table 1. List and properties of vinyl-terminated polydimethylsiloxanes and Methylhydromethylsiloxanes-Dimethylsiloxane copolymers

9. Table 2. Physical Properties of PDMS mixtures. 
Vinyl Terminated PolyDimethylsiloxanes

MW: 6000-140,000 (g/mol)<smiles>[X]C(C)(C)O[Si](C)(C)O[Si](C)(C)C=C</smiles>

Hydrosilylation
MethylHydrosiloxane - Dimethylsiloxane

Copolymer

MW: 22,5000 (g/mol)<smiles>C[Si](C)(C)O[Si](C)(C)O[Si](C)(C)O[Si](C)(C)O[Si](C)(C)C</smiles>

Hydride (MeHSiO)

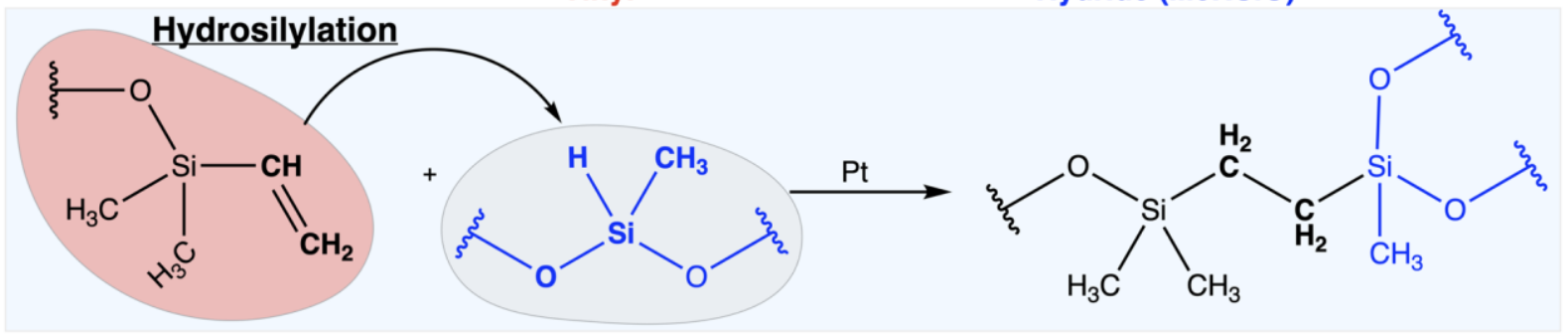

Figure 1. Hydrosilylation Reaction. Chemical structure of the Vinyl-Terminated PolyDimethylsiloxanes and the MethylHydrosiloxane-Dimethylsiloxane copolymer used as the bases and the crosslinkers of the novel PDMS membranes. The membranes were synthetized through a hydrosilylation reaction between the vinyl and the hydride (MeHSiO) in the presence of a platinum catalyst. 

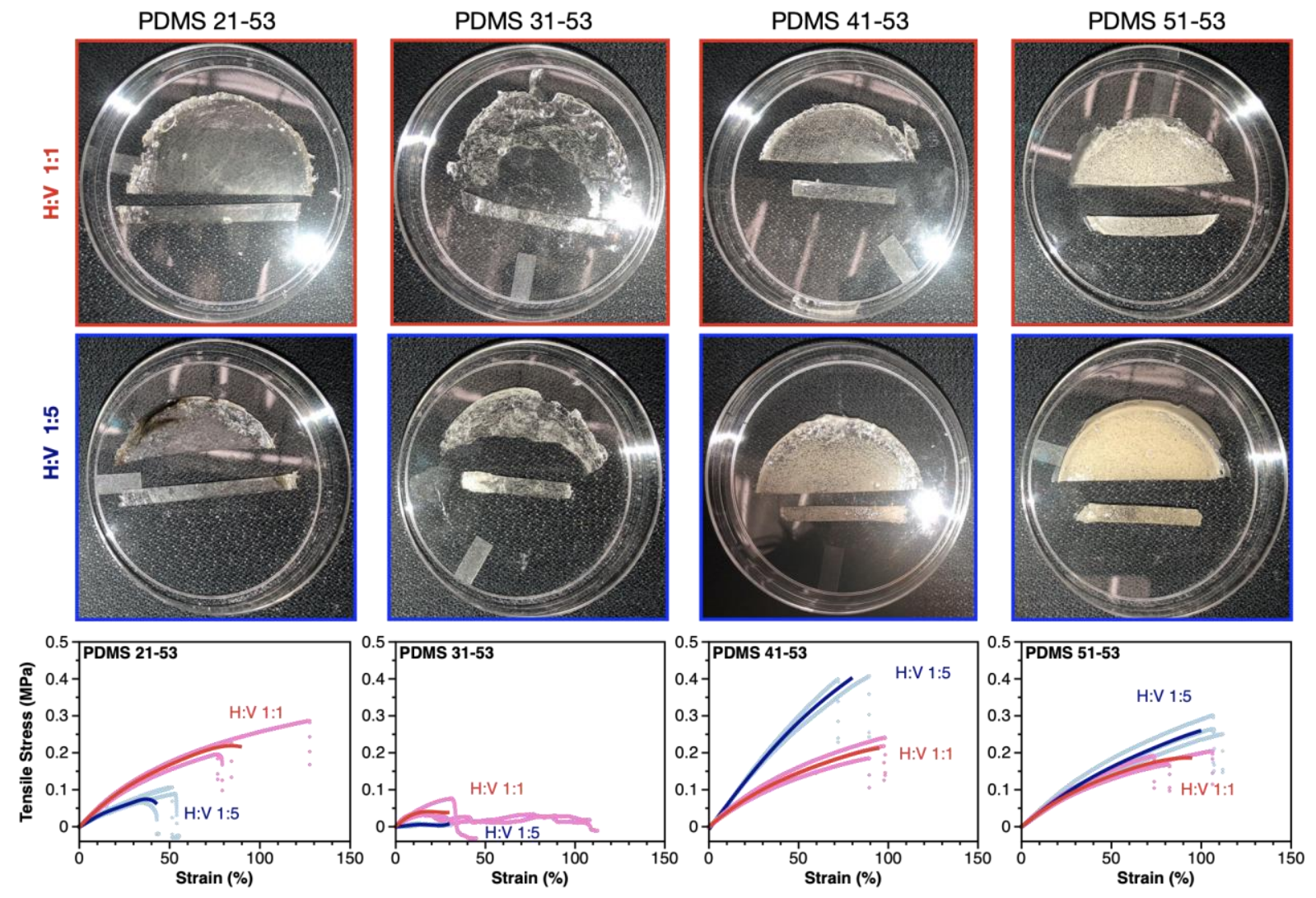

Figure 2. Mechanical Properties of the Novel PDMS Membranes. Commercially available vinyls, including DMS-V21, DMS-V31, DMS-V41, and DMS-V51, and a hydride (HMS053) mixed at ratio of 1:1 and 1:5 to generate PDMS 21-53, PDMS 31-53, PDMS 41-53, PDMS 51-53 membranes, respectively. The tensile properties of new membranes were measured at a strain rate of $0.15 \mathrm{~min}^{-1}$. The average tensile strength is presented for each mixing ratio and combination. 

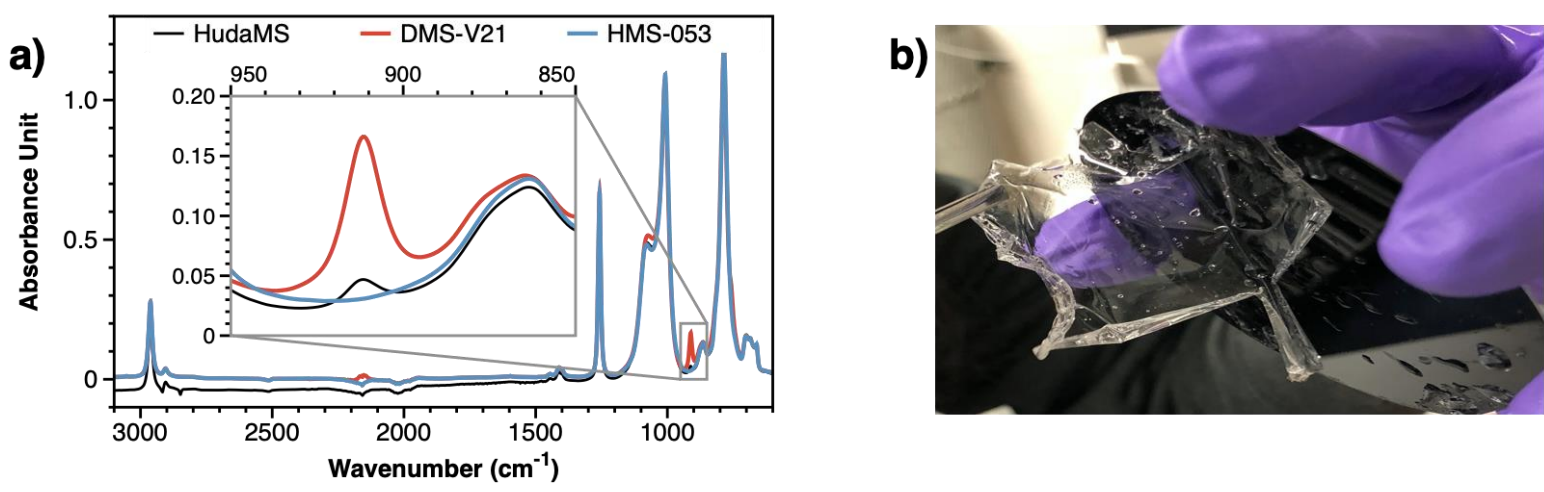

c)
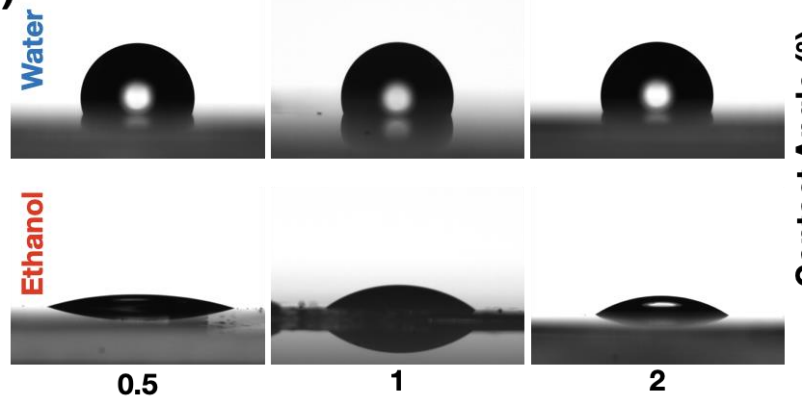

Hydride/Vinyl Ratio

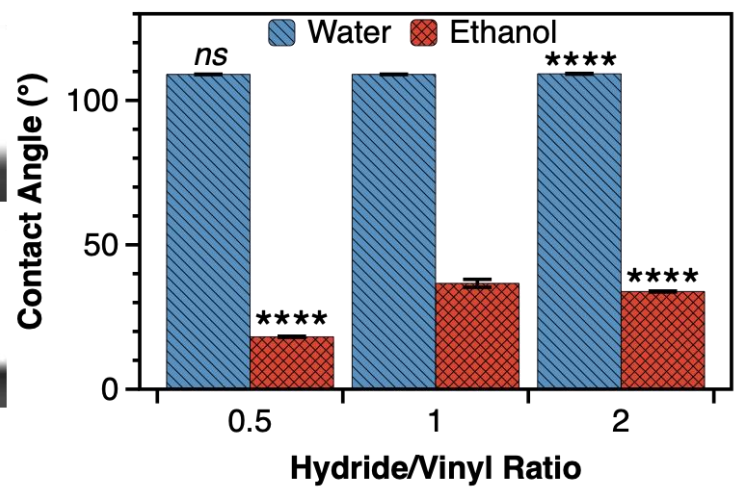

Figure 3. Characterization of new PDMS Membranes. (a) FTIR spectra were collected for the crosslinker (hydride), the base (vinyl) and the PDMS membranes. Peak at $\sim 910 \mathrm{~cm}^{-1}$ refers to the vinyl carbon-carbon double bonds, which become saturated during hydrosilylation reaction and crosslinking process. (b) Spin-coating of PDMS mixture onto silicon wafer at 300-500 RPM generates transparent membranes with thicknesses ranging between 21-140 $\mu \mathrm{m}$. (c) Water and ethanol contact angles were measured for a $2 \mathrm{~nL}$ droplet on PDMS membranes made with varying functional group concentrations. 

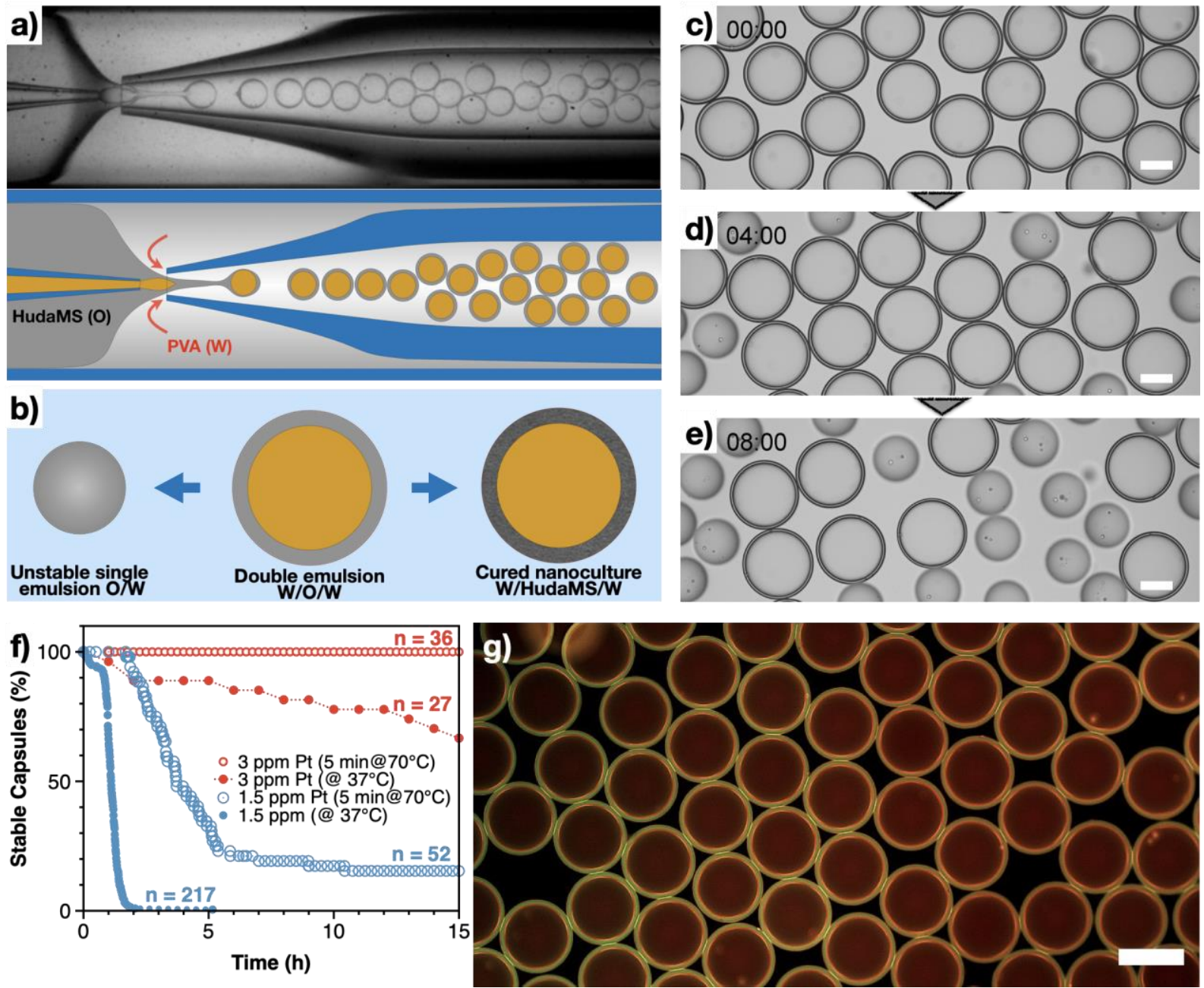

Figure 4. Generation of PDMS Microcapsules using a Flow-Focusing Microfluidic Device. (a) The three liquid phases are introduced into the device and directed to a 3-phase interface for high-throughput generation of monodisperse w/o/w double-emulsions. As illustrated, the aqueous culture medium, the PDMS mixtures, and the PVA solution constitute the 3 phases involved in the $\mathrm{w} / \mathrm{o} / \mathrm{w}$ double emulsions. (b) Mechanical instability converts double emulsions into single emulsions. Successful crosslinking of PDMS membranes leads to stable microcapsules. (c-e) Uncured PDMS capsules become unstable and burst into single emulsions. Scale bar: $50 \mu \mathrm{m}$ (f) PDMS microcapsule stability is improved with the addition of Pt catalyst and heat pre-treatments. (g) Stable PDMS microcapsules are achieved by mixing the hydride and vinyl at a 0.6:1 ratio, supplementing the mixture with $3 \mathrm{ppm}$ Pt catalyst and heatpretreatment at $70^{\circ} \mathrm{C}$ for $5 \mathrm{~min}$. Scale bar: $150 \mu \mathrm{m}$. 

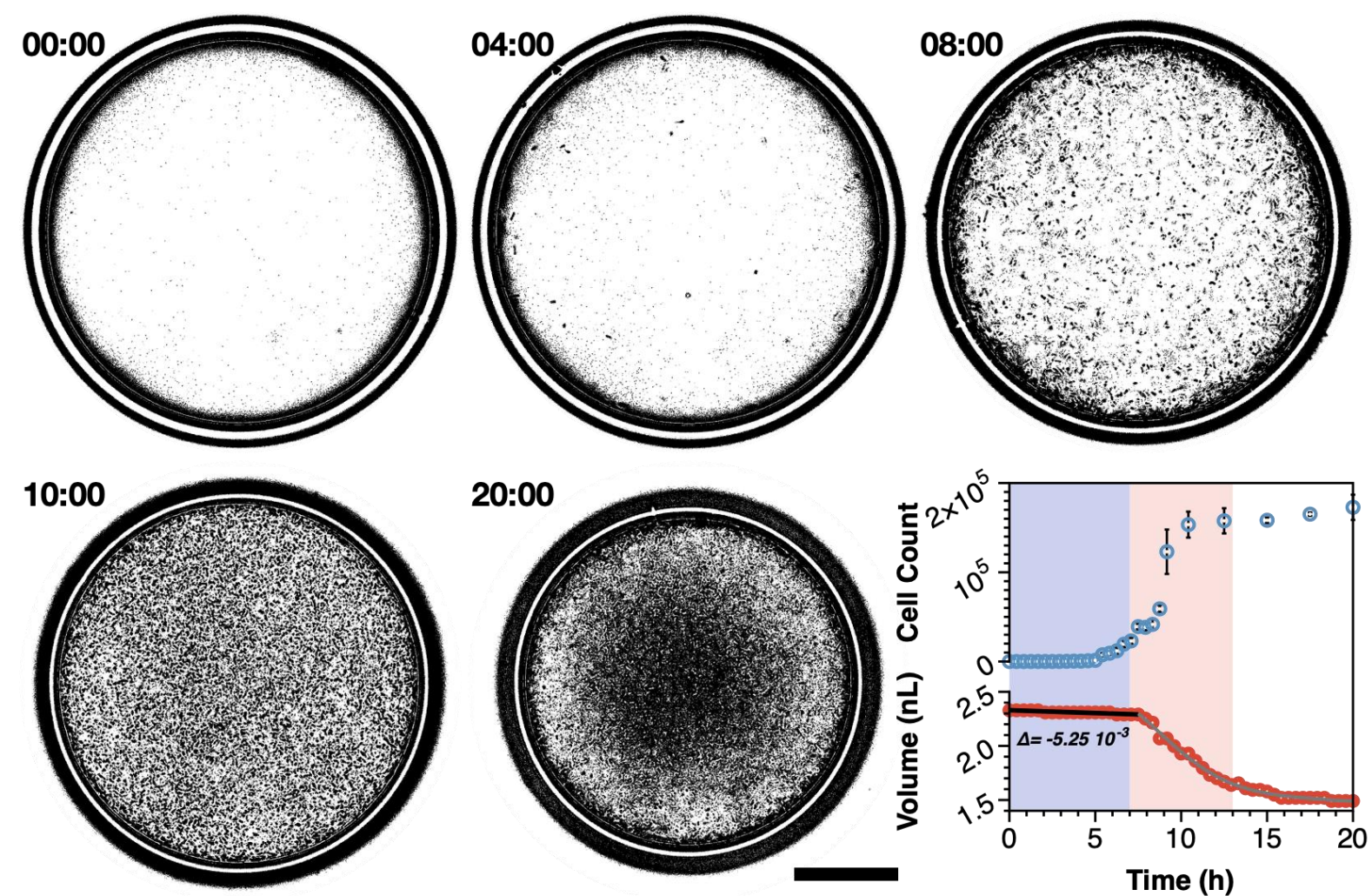

Figure 5. Growth Dynamics of PAO1 Cells in the Nanocultures. Grayscale rendering of the PDMS nanoculture recorded for $20 \mathrm{~h}$ displays a confluent growth of Pseudomonas aeruginosa. The exponential growth stage is achieved between $7-13 \mathrm{~h}$ and the stationary phase is attained after $13 \mathrm{~h}$ of incubation. Incubation of PAO1 nanocultures in $154 \mathrm{mM} \mathrm{NaCl}$ results in a sharp decrease in the size of the microcapsules. Scale bar: $50 \mu \mathrm{m}$. 
a)
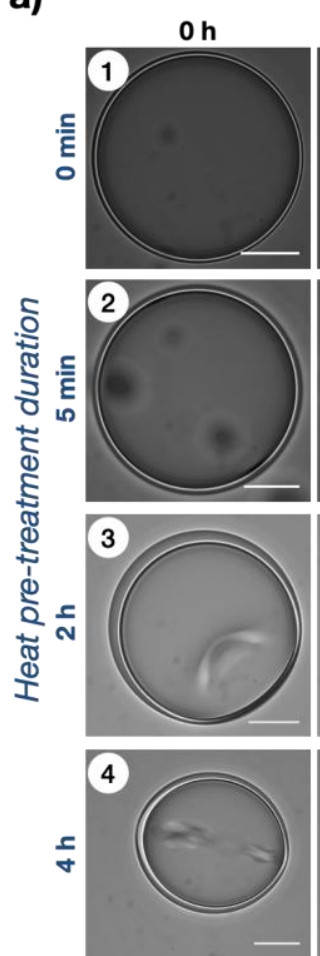

Osmotic annealing time
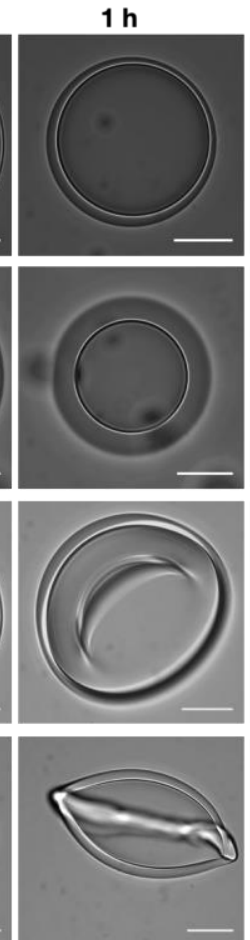
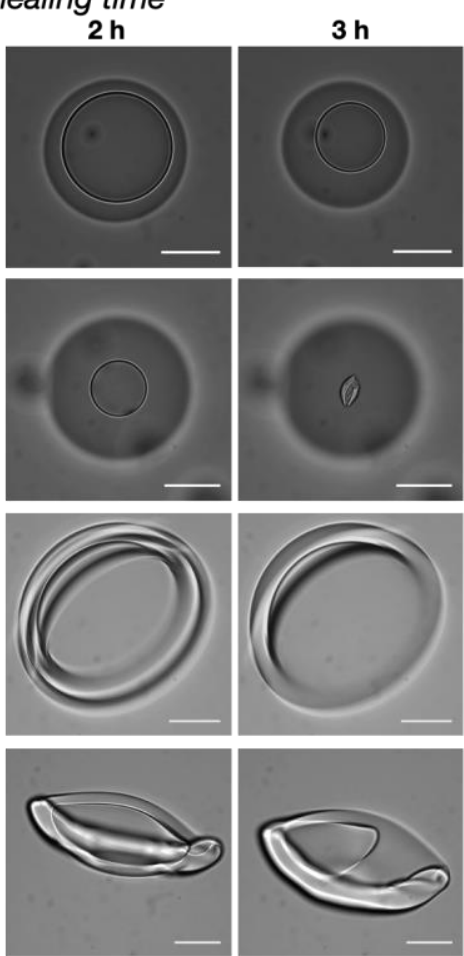

b)
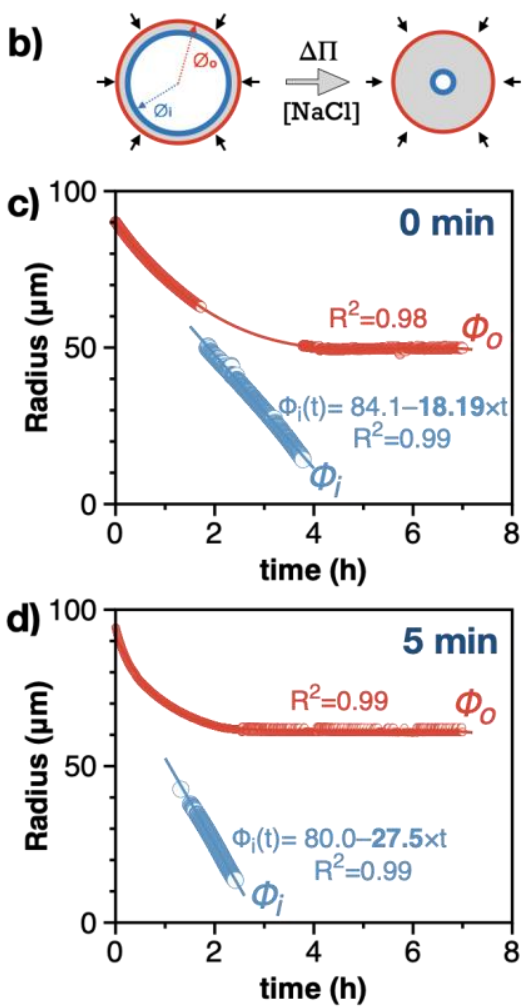

Figure 6. Transport and Mechanical Properties of PDMS Microcapsules. The microcapsules were subjected to osmotic pressure in a $10 \% \mathrm{NaCl}$ solution for 7 hours, to determine the mechanical stability of the capsules after heat treatment. (a) Osmotic annealing induced shrinkage of uncured (Panel $1 \&$ 2) and buckling of cured (Panel 3 \& 4) PDMS microcapsules. Polymerization of PDMS membrane is initiated after heat exposure at $70^{\circ} \mathrm{C}$ for $5 \mathrm{~min}$ as seen with the solid interface in Panel 2. Scale bar: 50 $\mu \mathrm{m}$. (b) Schematic illustration of the osmotically-induced shape deformation of the microcapsules. The inner $\left(\Phi_{i}\right)$ and outer $\left(\Phi_{o}\right)$ radii of the microcapsules change as a function of time. Radial change in the shrinking microcapsules is compared before (c) and after (d) heat pre-treatment at $70^{\circ} \mathrm{C}$ for $5 \mathrm{~min}$. The slope of the fit for the radial evolution of the inner droplet describes the radial velocity of the water flux during osmosis. 

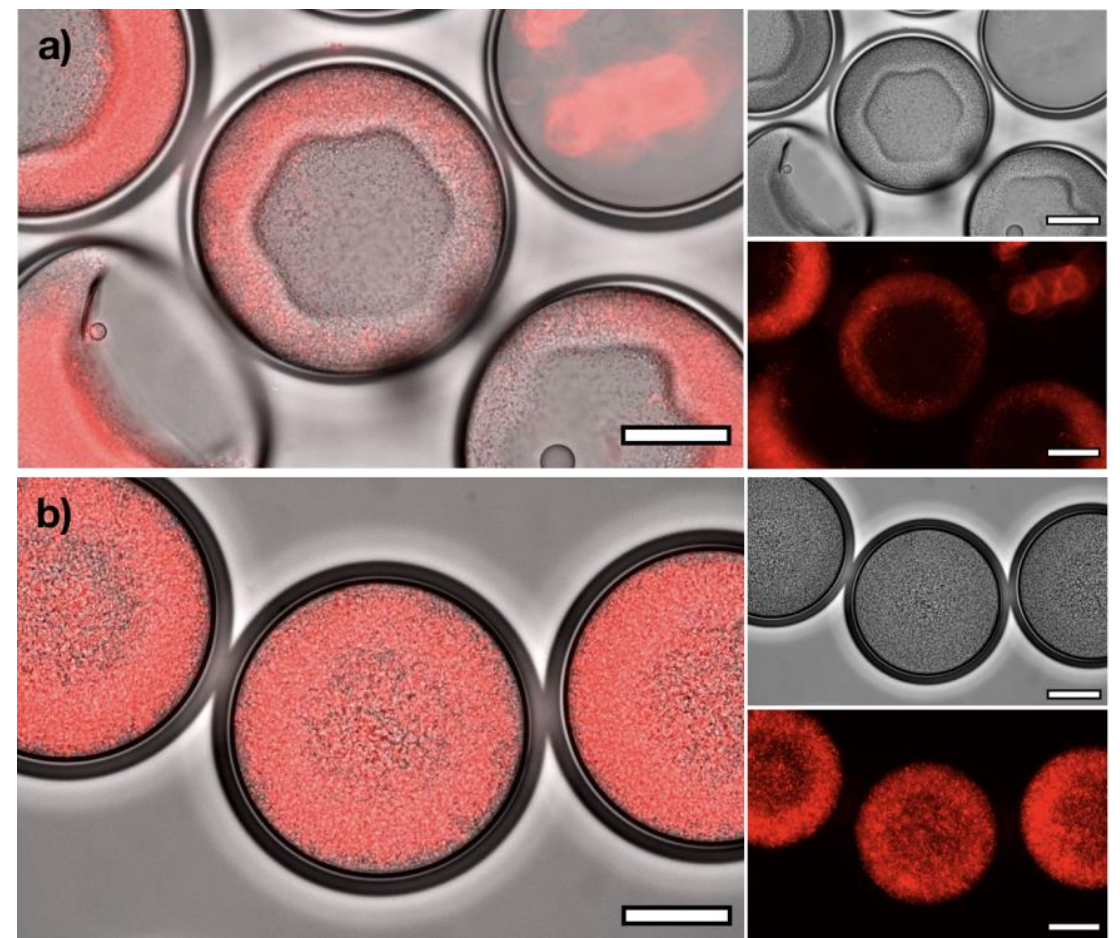

c)
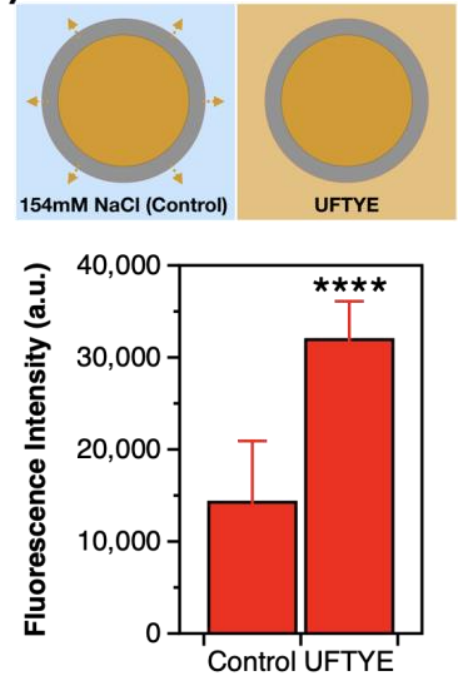

Figure 7. Transport Process Associated with PDMS Nanocultures. PDMS nanocultures containing a RFP-labelled E. coli suspension in UFTYE were incubated in (a) hypotonic (154 mM NaCl solution) vs. (b) isotonic (UFTYE) conditions. While incubation in the hypertonic condition lead to buckling of the crosslinked polymeric shell, the nanoculture grown in isotonic condition maintained their mechanical integrity. (c) Fluorescent imaging demonstrates that a high cell density is achieved in an isotonic condition. Scale bar: $50 \mu \mathrm{m}$. 
Table 1. List and properties of vinyl-terminated PDMS and MethylhydromethylsiloxanesDimethylsiloxane copolymers.

\begin{tabular}{|l|c|c|c|}
\hline & MW (g/mol) & $\begin{array}{c}\text { Wgt\% Vinyl / } \\
\text { Mol \% MeHSiO }\end{array}$ & Viscosity \\
\hline \multicolumn{3}{|c|}{ Vinyls } \\
\hline DMSV21 & 6000 & $0.8-1.2$ & 100 \\
\hline DMSV31 & 28000 & $0.18-0.26$ & 1000 \\
\hline DMSV41 & 62700 & $0.08-0.12$ & 10000 \\
\hline DMSV51 & 140000 & $0.03-0.04$ & 100000 \\
\hline \multicolumn{4}{|c|}{ Hydrides } \\
\hline HMS053 & $22,000-25,000$ & $4-6$ & $750-1000$ \\
\hline
\end{tabular}

Table 2. Physical Properties of PDMS mixtures.

\begin{tabular}{|l|c|c|c|}
\hline Mixtures & Viscosity & $\begin{array}{c}\text { Elastic } \\
\text { Modulus } \\
\text { ( H:V 1:1) }\end{array}$ & $\begin{array}{c}\text { Elastic } \\
\text { Modulus } \\
\text { ( H:V 1:5) }\end{array}$ \\
\hline PDMS 21-53 & 240 & $48.1 \pm 3.3$ & $28.8 \pm 2.1$ \\
\hline PDMS 31-53 & 990 & $34.3 \pm 8.2$ & $4.5 \pm 4.5$ \\
\hline PDMS 41-53 & 9280 & $40.3 \pm 4.1$ & $60.0 \pm 1.1$ \\
\hline PDMS 51-53 & 2650 & $38.1 \pm 4.6$ & $39.8 \pm 5.8$ \\
\hline
\end{tabular}

Keyword: poly(dimethylsiloxane) membranes, microfluidics, microbial cultures, growth dynamics

Huda Usman, Shanna Davidson, Nithil Manimaran, Jenna T. Nguyen, Aïssatou Bah, Rishabh Seth, Eric Beckman, Tagbo H.R. Niepa* 
Design of a novel Poly(dimethylsiloxane)-based microbial nanoculture system

ToC figure
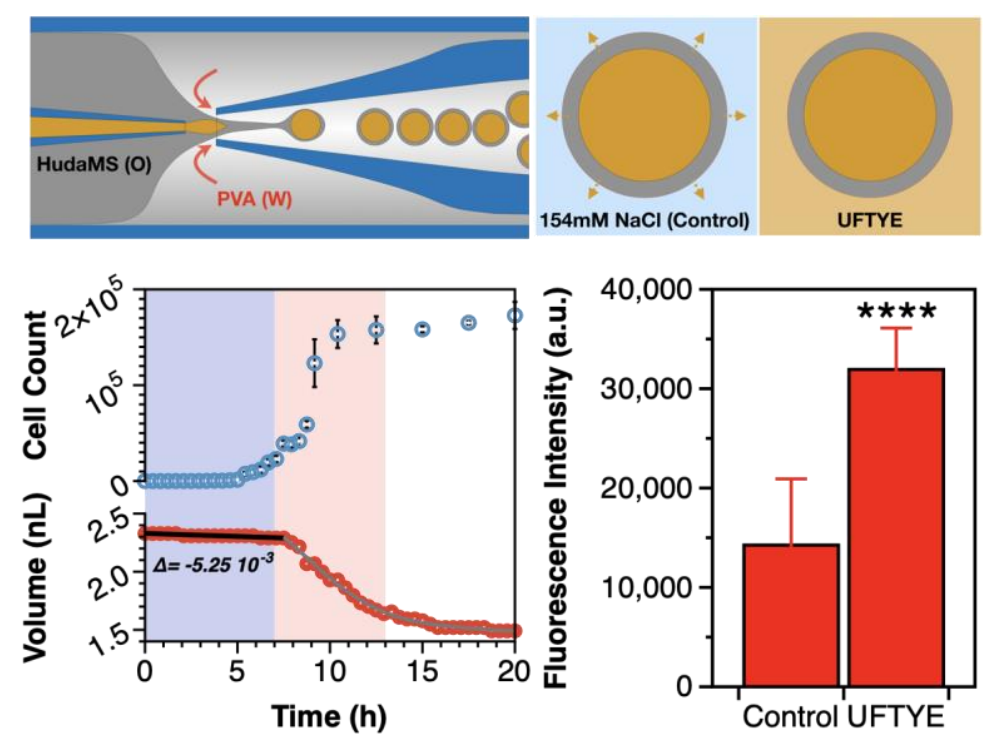


\title{
Supporting Information
}

\author{
Huda Usman, ${ }^{l}$ Shanna-Leigh Davidson, ${ }^{1}$ Nithil H. Manimaran, ${ }^{1}$ Jenna T. Nguyen, ${ }^{1}$ Aïssatou Bah, ${ }^{1}$ \\ Rishabh Seth, ${ }^{2}$ Eric Beckman, ${ }^{I}$ Tagbo H.R. Niepa ${ }^{1,2,3,4,5 *}$
}

${ }^{1}$ Department of Chemical and Petroleum Engineering, ${ }^{2}$ Department of Bioengineering, ${ }^{3}$ Department of Civil and Environmental Engineering, ${ }^{4}$ Department of Mechanical Engineering and Materials Science, ${ }^{5}$ Center for Medicine and the Microbiome, University of Pittsburgh, Pittsburgh, PA 15261, United States

\section{E-mail: tniepa@pitt.edu}

Keywords: poly(dimethylsiloxane) membranes, microfluidics, microbial cultures, biofilm, growth dynamics, microbial community

SV 1. Stability of the untreated PDMS nanocultures. Scale bar: $150 \mu \mathrm{m}$ (MOV).

SV 2. Growth dynamics of PAO1 cells in PDMS nanocultures. Scale bar: $25 \mu \mathrm{m}$ (MOV).

SV 3. Osmotic annealing of PDMS microcapsules treated at $70^{\circ} \mathrm{C}$ for 0 min. Scale bar: $50 \mu \mathrm{m}(\mathrm{MOV})$.

SV 4. Osmotic annealing of PDMS microcapsules treated at $70^{\circ} \mathrm{C}$ for $5 \mathrm{~min}$. Scale bar: $50 \mu \mathrm{m}(\mathrm{MOV})$.

SV 5. Osmotic annealing of PDMS microcapsules treated at $70^{\circ} \mathrm{C}$ for $2 \mathrm{~h}$. Scale bar: $50 \mu \mathrm{m}$ (MOV).

SV 6. Osmotic annealing of PDMS microcapsules treated at $70^{\circ} \mathrm{C}$ for $4 \mathrm{~h}$. Scale bar: $50 \mu \mathrm{m}(\mathrm{MOV})$.

SV 7. Osmotic annealing of Stable Microcapsules at 24h incubation. Scale bar: $150 \mu \mathrm{m}$ (MOV)

SV 8. Z-stack images of RFP-labelled E. coli Nanoculture (hypertonic growth). Scale bar: $50 \mu \mathrm{m}$ (MOV).

SV 9. Z-stack images of RFP-labelled E. coli Nanoculture (isotonic growth). Scale bar: $50 \mu \mathrm{m}$ (MOV). 
Click here to access/download Video

SV 1-Stability of the untreated HudaMS nanocultures15h.mov 
Click here to access/download Video

SV 2. Growth dynamics of PAO1 cells in HudaMS nanocultures.mov 
Click here to access/download Video

SV 3-Osmotic annealing of microcapsules at $70 \mathrm{C}$ for Omin.mov 
Click here to access/download Video SV 4-Osmotic annealing of microcapsules at $70 \mathrm{C}$ for 5min.mov 
Click here to access/download Video

SV 5-Osmotic annealing of microcapsule at $70 \mathrm{C}$ for 2h.mov 
Click here to access/download Video

SV 6-Osmotic annealing of microcapsules at 70C for 4h.mov 
Click here to access/download Video

SV 7-Osmotic annealing of Microcapsules at 24h incubation.mov 
Click here to access/download Video

SV 8-Z-stack images of RFP-E. coli Nanoculture_hypertonic.mov 
Click here to access/download Video

SV 9-Z-stack images of RFP-E. coli Nanoculture_isotonic.mov 